Not to appear in Nonlearned J., 45.

\title{
MULTIFREQUENCY POLARIMETRY OF THE NRAO 140 JET: POSSIBLE DETECTION OF A HELICAL MAGNETIC FIELD AND CONSTRAINTS ON ITS PITCH ANGLE
}

\author{
Keiichi Asada ${ }^{1,2}$, Makoto Inoue ${ }^{1}$, Masanori Nakamura ${ }^{3}$, Seiji Kameno ${ }^{1,4}$ and Hiroshi \\ Nagai $^{1,5}$ \\ National Astronomical Observatory of Japan, \\ 2-21-1 Osawa Mitaka Tokyo, 188-8588, Japan \\ asada@vsop.isas. jaxa.jp
}

\begin{abstract}
We present results from multifrequency polarimetry of NRAO 140 using the Very Long Baseline Array. These observations allow us to reveal the distributions of both the polarization position angle and the Faraday rotation measure (RM). These distributions are powerful tools to discern the projected and line-of-sight components of the magnetic field, respectively. We find a systematic gradient in the RM distribution, with its sign being opposite at either side of the jet with respect to the jet axis. The sign of the RM changes only with the direction of the magnetic field component along the line of sight, so this can be explained by the existence of helical magnetic components associated with the jet itself. We derive two constraints for the pitch angle of the helical magnetic field from the distributions of the RM and the projected magnetic field; the RM distribution indicates that the helical fields are tightly wound, while that of the projected magnetic field suggests they are loosely wound around the jet axis. This inconsistency may be explained if the Faraday rotator is not cospatial with the
\end{abstract}

\footnotetext{
${ }^{1}$ National Astronomical Observatory of Japan

${ }^{2}$ Institute of Space and Astronautial Science, Japan Aerospace Exploration Agency, 3-1-1 Yoshinodai, Sagamihara, Kanagawa, 229-8510, Japan

${ }^{3}$ Theoretical Astrophysics, Los Alamos National Laboratory

${ }^{4}$ Department of Physics, Faculty of Science, Kagoshima University

${ }^{5}$ Department of Astronomical Science, The Graduate University for Advanced Studies
} 
emitting region. Our results may point toward a physical picture in which an ultra-relativistic jet ("spine") with a loosely wound helical magnetic field is surrounded by a sub-relativistic wind layer ("sheath") with a tightly wound helical magnetic field.

Subject headings: galaxies: active — galaxies: jets — galaxies: quasars: individual (NRAO 140)

\section{Introduction}

Jets from active galactic nuclei (AGNs) maintain well-collimated structure and can travel for more than $100 \mathrm{kpc}$. It has come to be known that the bulk Lorentz factors of these jets reach $\Gamma=30$ in some cases (Kellermann et al. 2004). The mechanisms of formation, acceleration, and collimation of AGN jets are, however, still unclear. Magnetohydrodynamic (MHD) mechanisms are frequently invoked to model these jets. Magnetically driven outflows powered by a spinning black hole (Blandford \& Znajek 1977) or an accretion disk (Blandford \& Payne 1982) have been widely discussed in terms of both the acceleration and collimation. MHD outflows originating in the AGN core, particularly those with a strong toroidal field encircling the collimated flow, can exhibit efficient acceleration and collimation. This continues over a scale of $10^{3}-10^{4}$ Schwarzschild radii, and the terminal Lorentz factors of MHD outflows have reached values of 10-100 in recent numerical studies (Vlahakis \& Königl 2004; Fendt \& Ouyed 2004; McKinney 2006). These theoretical pictures are qualitatively consistent with some VLBI observations (e.g., Junor et al. 1999; Horiuchi et al. 2006). In the theoretical models, the toroidal magnetic field plays an important role in the acceleration (through the magnetic pressure gradient force) and the collimation (through the magnetic tension force). Thus, the detection of helically twisted magnetic components associated with such jets is a crucial key to confirming the MHD models with observations.

It is important to reveal the structure and strength of the magnetic fields in jets, although the available observational methods are limited. One powerful option is polarimetry. In the optically thin synchrotron emission from a nonrelativistic plasma, the polarization position angle (P.A.) is perpendicular to the projected direction of the magnetic field (the component perpendicular to the line of sight). In addition, the fractional polarization is closely related to the relative degree of ordering in the magnetic field. For this purpose, very long baseline polarimetry (VLBP) has been applied since the technique's early development. It has been reported that the P.A.'s tend to be parallel with respect to the jet axis for BL Lacertae objects whereas they tend to be perpendicular for quasars (Cawthorne et al. 1993). Jorstad et al. (2007), using multifrequency polarization observations, found a 
similar dichotomy but also suggested that it is not simply based on the optical classification of an object. The trend in BL Lac objects is sometimes explained by the compression due to strong shocks in the jets (Laing 1980; Hughes et al. 1989). Another possible explanation is that it is due to a toroidal magnetic field (Gabuzda et al. 2000) or the toroidal component of a helical magnetic field (Asada et al. 2002). However, the situation is not this simple, because the observed direction of the P.A. is not generally orthogonal to the projected direction of the magnetic field in the case of a relativistically moving, optically thin jet (Lyutikov et al. 2005). Neither is it simple to discriminate between these possibilities, since we have not taken advantage of the information derivable from the line-of-sight component of the magnetic field.

Further possibilities for examining the structure of such magnetic fields have been provided by the capability of full polarimetry with the Very Long Baseline Array (VLBA). The line-of-sight component of the magnetic field can be probed by the distribution of the Faraday rotation measure $(\mathrm{RM})$, since this is related to the electron density $n_{e}$ and the magnetic field component parallel to the line of sight $B_{\mathrm{LOS}}$ as $\mathrm{RM} \sim \int_{\mathrm{LOS}} n_{e} B_{\mathrm{LOS}} d r$, where $\int_{\mathrm{LOS}} d r$ represents integration along the line of sight. By combining the distributions of both the $\mathrm{RM}$ and the projected magnetic field, one can more reliably investigate the three-dimensional structure of the magnetic fields in parsec-scale jets.

The first inference of the presence of a helical magnetic field structure from the RM distribution was for the 3C 273 jet, based on 5-8 GHz VLBA observations (Asada et al. 2002). Those observations revealed a gradient of the RM across the jet, which can be interpreted as indicating the presence of the toroidal component of a helical field (Asada et al. 2002). In the simple case where we are seeing such a field from the side (a viewing angle of $90^{\circ}$ ), the sign of the line-of-sight component of the magnetic field will differ on the two sides of the jet as the field reverses direction. If the viewing angle decreases, the antisymmetric distribution will remain, with only an additional offset in the absolute value of the RM. Therefore, an RM gradient is expected in the presence of a helical magnetic field for arbitrary viewing angles, except $0^{\circ}$. After this initial work, a similar RM gradient in 3C 273 was confirmed by independent observations (Zavala \&Taylor 2005; Attridge et al. 2005; Asada et al. 2008). The same kinds of gradients have been reported for several BL Lac objects as well (Gabuzda et al. 2004).

In this paper we report observations of a bright quasar NRAO 140, which show an RM gradient across its jet, implying the presence of helical magnetic components. NRAO 140 is a distant quasar with a redshift, $z$, of 1 . 263. If we assume $H_{0}=73 \mathrm{~km} \mathrm{~s}^{-1} \mathrm{Mpc}^{-1}$ and $q_{0}=0.5,1$ mas corresponds to 8.4 pc. A value of 1 mas yr$^{-1}$ corresponds to $27.4 c$, and a superluminal motion of $11.0 c$ was reported with VLBA observations at $15 \mathrm{GHz}$ 
(Kellermann et al. 2004).

The paper is organized as follows: In $\S 2$, we describe the details of the VLBA polarimetry observations and calibrations. In $\S 3$, we present polarization images of NRAO 140 jet and summarize several properties of the projected magnetic field and the RM. Discussion and conclusions are given in $\S \S 4$ and 5 .

\section{Observations and Data Reductions}

The observations were carried out on 2003 January 13 using all 10 stations of the VLBA. We chose intermediate frequencies ( IFs) of 4. 618, 4. 688, 4. 800, and 5. $093 \mathrm{GHz}$ in the 5 $\mathrm{GHz}$ band and 8. 118, 8. 188, 8. 402, and 8. $593 \mathrm{GHz}$ in the $8 \mathrm{GHz}$ band. Each IF has an $8 \mathrm{MHz}$ bandwidth. Both left- and right-circular polarizations were recorded at each station. The integration time was 55 minutes in each frequency band at various hour angles. We observed 3C 84 as a calibration source for the instrumental polarization and DA 193 for the polarization position angle. Calibrations were performed in the same manner as for our RM observations toward 3C 273, which are described in Asada et al. (2008).

The instrumental polarizations of the antennas are determined for each IF at each band with 3C 84, using an AIPS task LPCAL. The polarization-angle offset at each station was calibrated using DA 193 obtained by VLA/VLBA Polarization Calibration Monitoring Program (Myers \& Taylor). The source was observed on 2002 Dec. 17 with VLA at 4.8851 and 4. $8351 \mathrm{GHz}$ and 8. 4351 and 8. $4851 \mathrm{GHz}$. Total flux of DA 193 measured with VLA is $5.70 \pm 0$. $01 \mathrm{Jy}$ at $5 \mathrm{GHz}$ and $4.98 \pm 0$. $01 \mathrm{Jy}$ at $8 \mathrm{GHz}$, while those measured with VLBA are, in average over four IFs, 5. $42 \pm 0.24 \mathrm{Jy}$ and $5.01 \pm 0.21 \mathrm{Jy}$, respectively. In addition, total polarized flux measured with VLA is $55.0 \pm 2.7$ mJy at $5 \mathrm{GHz}$ and 73 . $1 \pm 0$. $1 \mathrm{mJy}$ at $8 \mathrm{GHz}$, while those measured with VLBA are, in average over four IFs, $55 \pm 15 \mathrm{mJy}$ and $81 \pm 3 \mathrm{mJy}$, respectively. Thus the time variation between our and VLA measurement would be small, and our calibration would be reasonable.

Observing frequencies are slightly different between VLA and VLBA observations. We evaluated RM of DA 193 with VLA measurements, and we estimated PAs for each IFs by interpolation. In order to obtain the distributions of RM and projected magnetic field, we restored images at higher frequencies to match the resolution at the lowest frequency observation. The restored beam size was 2 . 79 mas $\times 1$. 28 mas with the major axis at a position angle of $0.25^{\circ}$. The distribution of RM was obtained by an AIPS task RM with polarization images at 4. 618, 5. 093, 8. 118, and 8. $593 \mathrm{GHz}$, with regions where the polarized intensity is three times greater than the r.m.s. noise in the polarized intensity. 


\section{Results}

The P.A. distributions at 4. 618 and 8. $118 \mathrm{GHz}$ are shown superposed on the distribution of total intensity in Figure 1. The core is presumably located at the northwest end of the jet, which extends to the southeast. The jet bends slightly toward the south at 10 mas from the core. We do not detect polarized flux from the core, at levels below $0.3 \%$ at 5 and $8 \mathrm{GHz}$. For the jet components, we detect significant polarized flux, at the $10 \%$ level. The P.A.'s in the jet are nearly perpendicular to the jet axis but slightly reflect the curvature across the jet. The curvature is greater at $5 \mathrm{GHz}$ than at $8 \mathrm{GHz}$. The difference in P.A.'s at the two frequencies can be explained by Faraday rotation, since the amount of rotation of the P.A. corresponds to $\lambda^{2}$. Therefore, nonuniform P.A. distributions result in a nonuniform RM distribution.

The distributions of the projected magnetic field and RM are shown superposed on the total intensity distribution at 4. $618 \mathrm{GHz}$ in Figure 2. The projected magnetic field runs roughly parallel to the jet axis, which is consistent with the usual trend seen in quasar jets (Cawthorne et al. 1993). Similarly to the case of the parsec-scale 3C 273 jet (Asada et al. 2002), we clearly see a gradient across the jet in the distribution of RM. In Figure 3, we show a cross section of the RM distribution along the line A-B from Figure 2, which is perpendicular to the jet axis. The RMs differ by as much as $150 \mathrm{rad} \mathrm{m}^{2}$, with an error less than $40 \mathrm{rad} \mathrm{m}^{2}$ and typically $20 \mathrm{rad} \mathrm{m}^{2}$. The sign of the RM is positive at the southeast side of the jet and negative at the northwest side. The sign of the RM only depends on the direction of the line-of-sight component of the magnetic field. This change is naturally interpreted as being due to a reversal of this component. We note that similar reversals of RM sign on opposite jet sides have also been reported for QSOs 0745+241 and 1652+398 (Gabuzda et al. 2004) and 3C 273 (Zavala \& Taylor 2005).

The RM gradient is detected out to 8 mas from the core, or $67 \mathrm{pc}$ in projected distance. The viewing angle of the jet can be constrained from the Doppler effect as $\theta_{\max }=$ $2 \arctan \left(1 / \beta_{\text {app }}\right)$, where $\theta_{\max }$ is the upper limit on the angle between the jet axis and the line of sight. Since an apparent motion $\beta_{\text {app }}$ of $11 \mathrm{c}$ was detected from previous VLBA observations ( Kellermann et al. 2004), this then gives an upper limit for the viewing angle of $10.4^{\circ}$. Therefore, the linear extent of the jet is greater than $350 \mathrm{pc}$, taking into account $\theta_{\max }$ $\sim 10.4^{\circ}$. 


\section{Discussion}

\subsection{Implications of the RM distribution}

The RM gradient can be simply explained as the result of a helical magnetic field, as has been discussed for many AGN jets (Asada et al. 2002, 2008; Gabuzda et al. 2004; Zavala \& Taylor 2005; Attridge et al. 2005). Similarly to $3 \mathrm{C} 273$, we can estimate the direction of the toroidal component. The RM on the northeast side of the jet is positive, so the line-of-sight component of the magnetic field is directed toward us. On the other hand, that on the southwest side is negative, and the line-of-sight component of the magnetic field is directed away from us. Therefore, the field twists counterclockwise as seen from the core looking downstream through the jet. In the case of $3 \mathrm{C} 273$, we discussed the possibility of detecting the longitudinal component of the helical magnetic field by using the offset of the RM (Asada et al. 2002). By combining the direction of the RM gradient and the estimated direction of the longitudinal component, we concluded that the orientation of $3 \mathrm{C}$ 273's helical field is that of a right-handed screw, reflecting the direction of rotation of the accretion disk or spinning black hole itself under the assumption that the helical magnetic field is wound up by the rotation. However, in NRAO 140 we could not detect any significant offset of the RM in its gradient. In the innermost regions of the jet, the RM is biased to the negative. If we assume that the offset can be ascribed to the longitudinal component of the helical magnetic field, we can uniquely predict the direction of the twisting of the helical magnetic field as being right-handed. If this field is wound by the rotation of the spinning black hole or the accretion disk, as suggested by the electromagnetic/MHD models (Blandford \& Znajek 1977; Blandford \& Payne 1982), it would appear that the northeastern half of the disk is approaching us and the southwestern half is receding. We identify the rotation direction of the accretion disk or black hole as clockwise as we see it, taking into account the configuration of the approaching jet.

\subsection{Constraint for the pitch angle of the helical magnetic field}

The RM gradient is a function of the pitch angle of the helical magnetic field and the viewing angle. Let us consider an observer simply seeing the field at a viewing angle of $\theta$. The line-of-sight component of the helical magnetic field, $B_{\mathrm{LOS}}$, can be expressed as follows:

$$
B_{\mathrm{LOS}}=B_{\psi} \sin \theta \sin \psi+B_{z} \cos \theta
$$

where $B_{\psi}$ and $B_{z}$ are the toroidal and longitudinal component of the helical magnetic field and $\psi$ is the azimuthal angle, varying from $-90^{\circ}$ to $90^{\circ}$. We show the line-of-sight component 
of the helical magnetic field across the jet at several pitch angles in Figure 4, assuming a viewing angle of $10.4^{\circ}$, which is the same as the upper limit derived from the Doppler analysis. The sign of the line-of-sight component on one side is opposite to that on the other side when the pitch angle is smaller than $\theta$, while the signs are identical when the pitch angle is larger than $\theta$. The threshold for having both signs in the line-of-sight component is

$$
B_{\psi} \sin \theta>B_{z} \cos \theta .
$$

In the case of the NRAO 140 jet, we detect both positive and negative signs in the RM gradient. In addition, we derived an upper limit on the viewing angle, $\theta_{\max }=10.4^{\circ}$ from Doppler analysis. Thus, we can place a constraint on the pitch angle of the helical magnetic field in order to have both positive and negative signs in the RM gradient, since the sign of the RM can be changed by the direction of the line-of-sight component of the field. If we define the pitch angle of the helical magnetic field, $\chi$, as $\tan \chi=B_{z} / B_{\psi}$,

$$
\tan \chi<\tan \theta<\tan \theta_{\max } .
$$

This gives an upper limit for the pitch angle of the helical magnetic field of $10.4^{\circ}$.

On the other hand, a constraint on the pitch angle can also be derived from the direction of the projected magnetic field. Let us consider a simple case in which we are looking from the side at a helical magnetic field in an optically thin jet, following Asada et al. (2002). For a large pitch angle, the magnetic field runs almost parallel to the jet, and the P.A.'s on both near and far sides of the jet are nearly perpendicular to the jet. Then the P.A. integrated across the jet is also perpendicular to the jet, so that the resultant direction of the projected magnetic field is expected to be almost parallel to the jet. If instead the pitch angle is small, the resultant direction of the projected magnetic field is expected to be almost perpendicular to the jet. Therefore, a dichotomy in the direction of the projected magnetic field is expected, due to the vector accumulation of the incoherent-polarization radiation (see, e.g., Asada et al. 2002). Taking into account the viewing angle, the constraint for the projected magnetic field to be parallel to the jet can be expressed as

$$
B_{z} \sin \theta>B_{\psi} .
$$

Again, if we define the pitch angle of the helical magnetic field by $\tan \chi=B_{z} / B_{\psi}$,

$$
\tan \chi>\frac{1}{\sin \theta}>\frac{1}{\sin \theta_{\max }} .
$$

This gives a lower limit for the pitch angle of the helical magnetic field of $79.8^{\circ}$. 
Thus, we have derived two constraints for the pitch angle of the helical magnetic field. One is $\chi<10.4^{\circ}$, and the other is $\chi>79.8^{\circ}$. These are obviously inconsistent, an inconsistency that may come from a difference between the region emitting high-energy particles and that corresponding to the Faraday rotation, that is, a Faraday screen. In fact, a reasonably strong fractional polarization of $10 \%$ is detected with an RM of $100 \mathrm{rad} \mathrm{m}^{2}$. For a simple slab model of internal Faraday rotation, the fractional polarization is expected to be smaller than $1.7 \%$ at $5 \mathrm{GHz}$ with an $\mathrm{RM}$ of $100 \mathrm{rad} \mathrm{m}^{2}$. Therefore, this would exclude internal Faraday rotation, and we suggest that a layer or sheath surrounding the emitting jet is responsible for the Faraday rotation (Inoue et al. 2003).

Such a spine-sheath structure has been strongly supported in several cases in terms of the amount of Faraday rotation being greater than $90^{\circ}$ ( Inoue et al. 2003; Gabuzda et al. 2004; Zavala \& Taylor 2005). A rapid time variation of the RM gradient in the 3C 273 jet also implies that MHD-driven AGN jets may have multiple, layered components (Asada et al. 2008). From a theoretical point of view, the spine may correspond to the ultrarelativistic outflows, with high Lorentz factors of $\Gamma \geq 10$. This idea originates in the fact that the electromagnetic (EM) energy strongly dominates the matter energy in the outflows produced by a spinning black hole (Blandford \& Znajek 1977). Thanks to the lack of matter inertia, the magnetic field has a large pitch angle (the local Alfvén speed may almost reach the speed of light, and an inertial back-reaction of the plasma on the poloidal field may not occur efficiently in the EM-dominated regions). This physical picture has been observed in recent general relativistic degenerate electrodynamic and MHD simulations (Komissarov 2001, 2004). However, the sheath may correspond to the sub-relativistic or mildly relativistic outflows, with low Lorentz factors of a few, and arise from the accretion disk (Tsinganos \& Bogovalov 2002). The matter can be accelerated by the Lorentz force, and the flows reach a superfast magnetosonic regime far from the disk. The flow may finally become matter dominated (kinetic plus internal energy), with a low pitch angle (Blandford \& Payne 1982; Vlahakis \& Kon̈igl 2004). Thus, a picture emerges in which an ultra-relativistic "jet" (the spine) with a loosely wound helical field, perhaps surrounded by mildly relativistic "winds" (the sheath) with a tightly wound helical field, may be a common structure in AGN jets, which is essentially consistent with our results.

On the other hand, in the case of M87 a Hubble Space Telescope (HST) observation provides us with angular resolution of 0.2 arcsec. This corresponds to a linear resolution of $15 \mathrm{pc}$, which is similar to that of our VLBA observations of NRAO 140. Based on this HST observation, Perlman et al. (1999) found that the M87 jet appears narrower in the optical than in the radio. This suggests that for arcsecond-scale jets, the jet consists of a highly relativistic spine and a lower velocity sheath; the highly relativistic spine may be responsible for the optical emission, and the lower velocity sheath may be responsible for 
the radio emission. If the slow sheath observed as an RM gradient exists in arcsecond-scale jets, this would indicate that there is another sheath wrapped around the highly relativistic spine and lower velocity sheath, suggesting that a stratified flow structure is more likely than a simple two-component model. A similar tendency has been reported for 3C 273 as well (Jester et al. 2005).

\section{Conclusions}

In order to discuss the three-dimensional configuration of the magnetic fields in parsecscale quasar jets, we have performed multifrequency VLBA polarimetry toward NRAO 140. We revealed the distributions of both the projected component of the magnetic field and the rotation measure. We find a systematic gradient in the RM distribution similar to that seen in the $3 \mathrm{C} 273$ jet. Furthermore, the sign of the RM is opposite on either side of the gradient. This is presumably due to a change of direction of the magnetic field and is naturally explained by a helical field structure. Using the properties of the RM gradient and the viewing angle, we derive a constraint for the pitch angle of the helical magnetic field in the layer or the sheath of the jet of $\chi<10.4^{\circ}$. On the other hand, from the direction of the projected magnetic field and the viewing angle, we derive another constraint for the pitch angle in the spine of the jet of $\chi>79.8^{\circ}$. Therefore, we expect that in NRAO 140 an ultra-relativistic jet (or "spine") with a loosely wound helical magnetic field is surrounded by a layer (or "sheath") with a tightly wound helical magnetic field.

Acknowledgments: The authors thank E. S. Perlman for a critical reading of this paper and valuable comments as a referee. The VLBA and VLA are operated by the National Radio Astronomy Observatory (NRAO), a facility of the National Science Foundation, operated under cooperative agreement by Associated Universities, Inc.

\section{REFERENCES}

Asada, K. , et al. 2002, PASJ, 54, 39L

Asada, K. , et al. 2008, ApJ, 675, 79

Attridge, J. M. , Wardle, J. F. C. , \& Homan, D. C. 2005, ApJL 633, 85

Blandford, R. D. \& Payne, D. G. 1982, MNRAS, 199, 883

Blandford, R. D. \& Znajek, R. L. 1977, MNRAS, 179, 433 
Cawthorne, T. V., Wardle, J. F. C. , Roberts, D. H. \& Gabuzda, D. C. 1993, ApJ, 416, 519

Fendt, C. \& Ouyed, R. 2004, ApJ, 608, 378

Gabuzda, D. C. , Murray, E. \& Cronin, P. 2004, MNRAS, 351, L89

Gabuzda, D. C. , Pushkarev, A. B. \& Cawthorne, T. V. 2000, MNRAS, 319, 1109

Horiuchi, S. , Meier, D. L. , Preston, R. A. , Tingay, S. J. 2006, PASJ, 58, 211

Hughes, P.A. , Aller, H. D. \& Aller, M. F. 1989, ApJ, 341, 54

Inoue, M. et al. 2003, Radio Astronomy at the Fringe, ASP Conference Proceedings, Vol. 300,141

Jester, S. , Röser, H. -J. , Meisenheimer, K. , Perley, R. 2005, A\&A, 431, 477

Jorstad, S. G. , et al. 2007, AJ, 134, 799

Junor, W. , Biretta, J. A. , \& Livio, M. 1999, Nature, 401, 891

Kellermann, K. I. , Lister, M. L. , Homan, D. C. , Vermeulen, R. C. , Cohen, M. H. , Ros, E. , Kadler, M. , Zensus, J. A. , \& Kovalev, Y. Y. 2004, ApJ, 609, 539

Komissarov, S. S. 2001, MNRAS, 326, L41

Komissarov, S. S. 2004, MNRAS, 350, 1431

Laing, R. A. 1980, MNRAS, 193, 439

Lyutikov, M. , Pariev, V. I. , \& Gabuzda, D. C. 2005, MNRAS, 360, 869

McKinney, J. C. 2006, MNRAS, 368, 1561

Perlman, E. S. , Biretta, J. A. , Zhou, F. , Sparks, W. B. , Macchetto, F. D. 1999, AJ, 117, 2185

Taylor, G. B. 1998, ApJ, 506, 637

Tsinganos, K. \& Bogovalov, S. 2002, MNRAS, 337, 553

Vlahakis, N. \& Königl, A. 2004 ApJ, 605, 656

Zavala, R. T. \& Taylor, G. B. 2005, ApJL 626, 73 

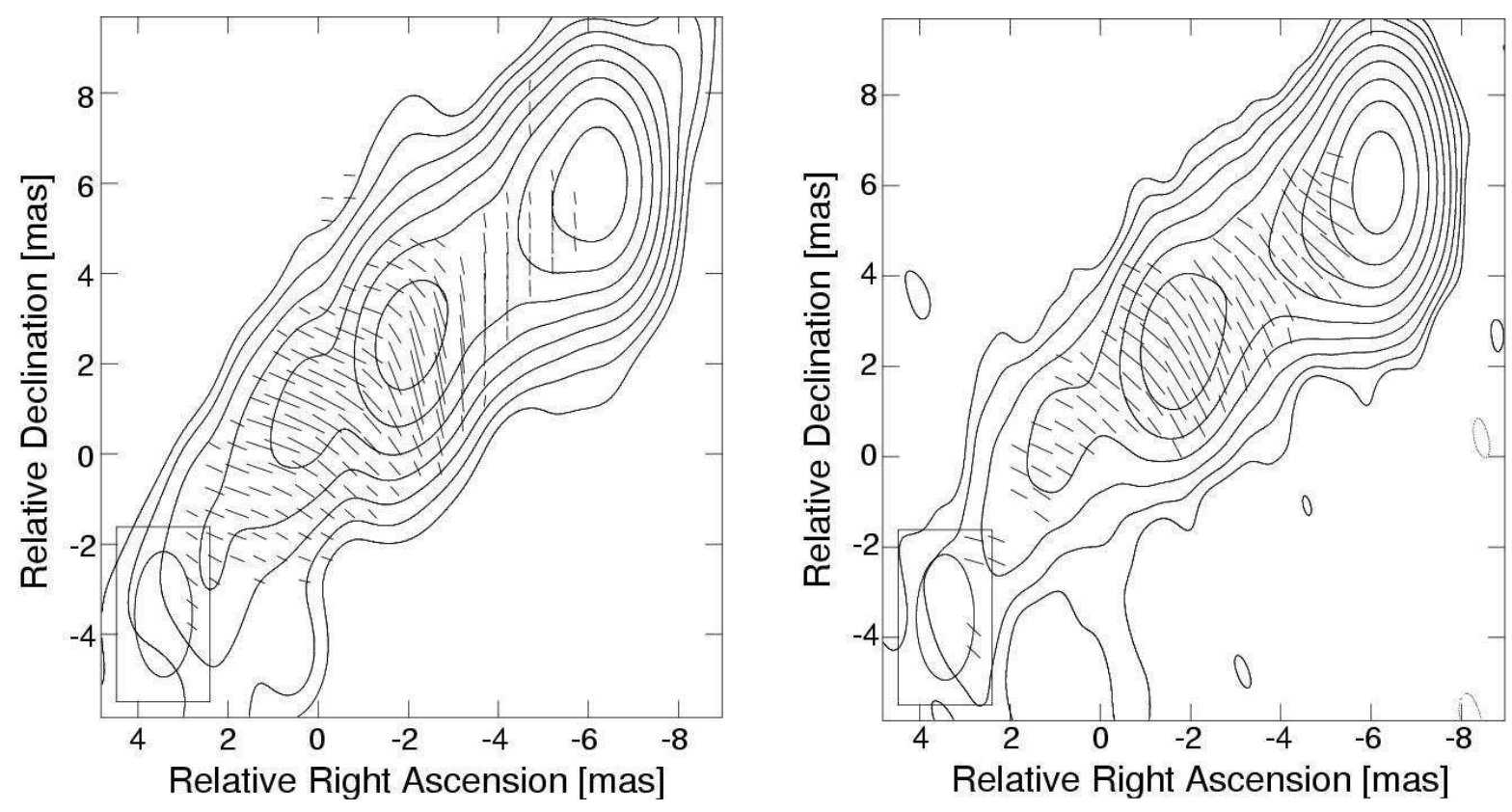

Fig. 1.- (left) Distribution of polarization position angle (bar) superposed on the contour images of the total intensity at $4.618 \mathrm{GHz}$. (right) Distribution of polarization position angle (bar) superposed on the contour images at $8.118 \mathrm{GHz}$. Contours are plotted at $-1,1$, $2,4,8,16,32,64,128,256,512$ and $1024 \times$ three times the $\mathrm{r}$. m. s. noise of that of the total intensity at each frequency. The synthesized beam size is restored by the $4.618 \mathrm{GHz}$ beam of 2.79 mas $\times 1.28$ mas with the major axis at a position angle of $0.25^{\circ}$. Image at 8. $118 \mathrm{GHz}$ is restored to the same resolution of $4.618 \mathrm{GHz}$. 

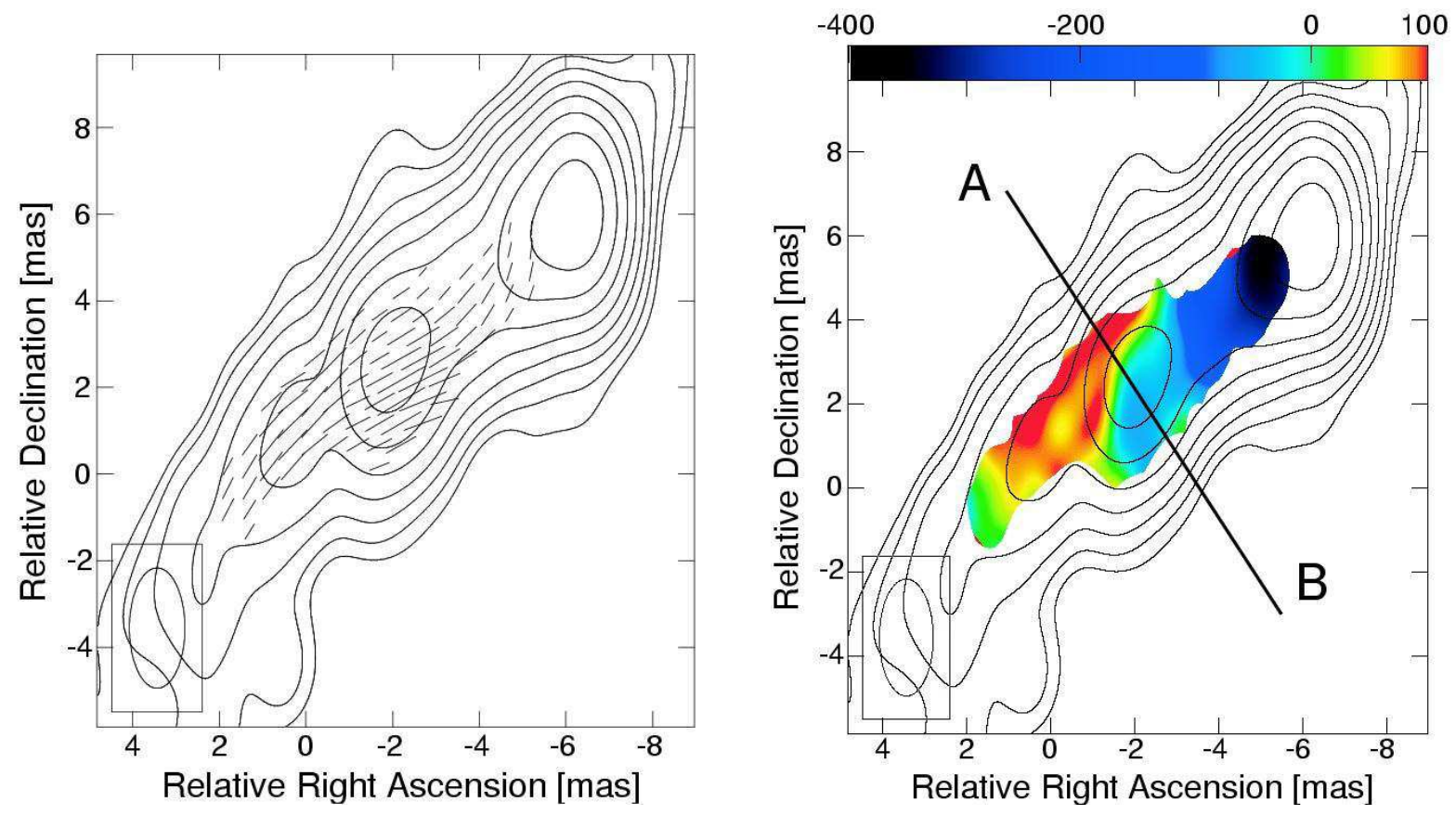

Fig. 2.- (left) Distribution of the projected component of the magnetic field (bar) superposed on the contour images of the total intensity at $4.618 \mathrm{GHz}$. Contours are plotted at $-1,1,2,4,8,16,32,64,128,256,512$ and $1024 \times$ three times the $\mathrm{r}$. m. s. noise of that of the total intensity at $4.618 \mathrm{GHz}$. The synthesized beam size is restored by the $4.618 \mathrm{GHz}$ beam of 2. 79 mas $\times 1.28$ mas with the major axis at a position angle of $0.25^{\circ}$. (right) Distribution of RM (color scale) superposed on the contour images of the total intensity at 4. $618 \mathrm{GHz}$. The RMs are plotted in the region where the polarized intensity is greater than three times the r. m. s. noise in the polarized intensity. 


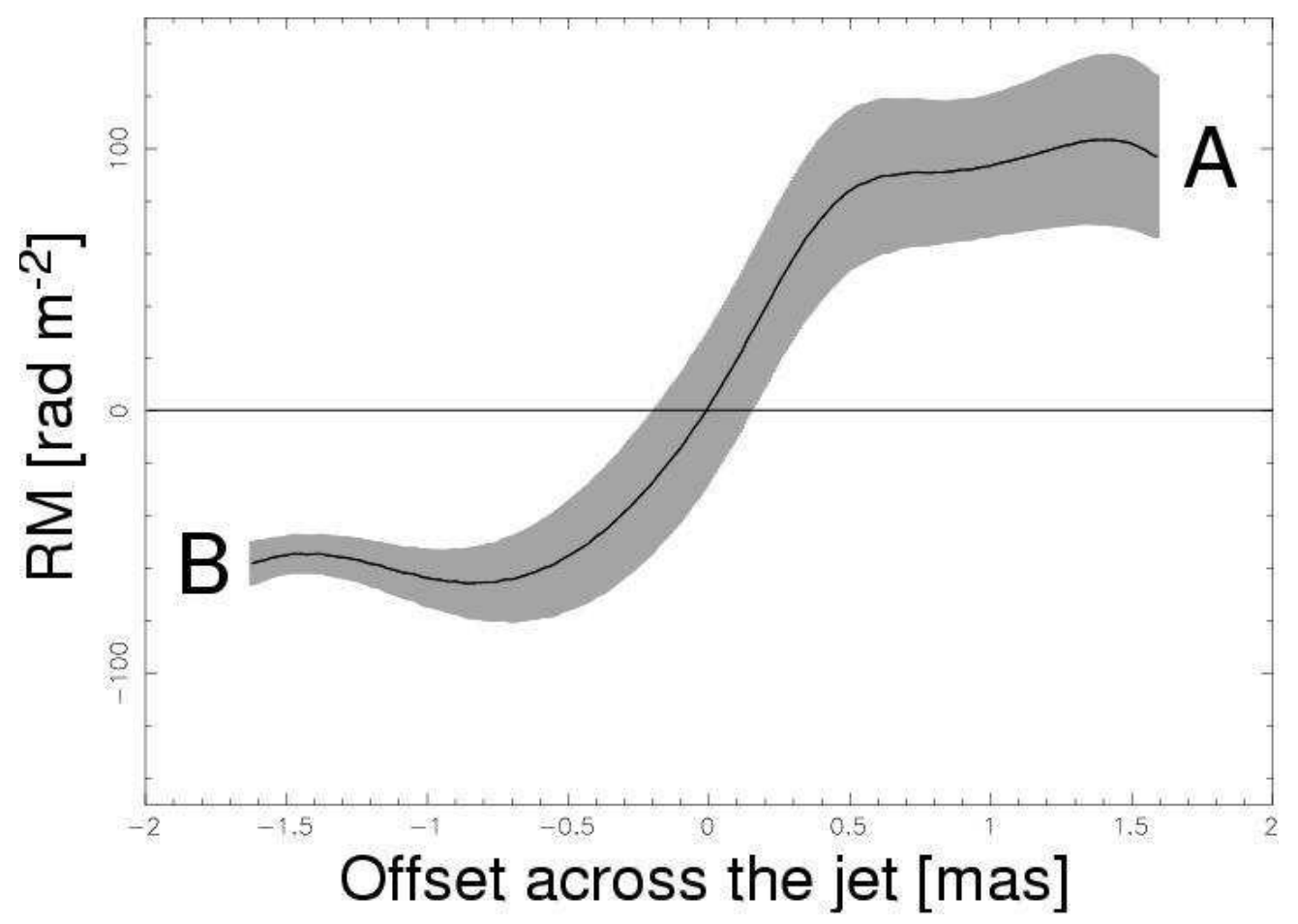

Fig. 3.- Cross sections of the RM distribution along the line $\mathrm{A}-\mathrm{B}$, derived using the AIPS task SLICE. The shaded area along the curved line of the RM indicates the standard deviation $(1 \sigma)$ in the RM. The profile of the RM distribution is anti-symmetric with respect to the central axis of the jet. 


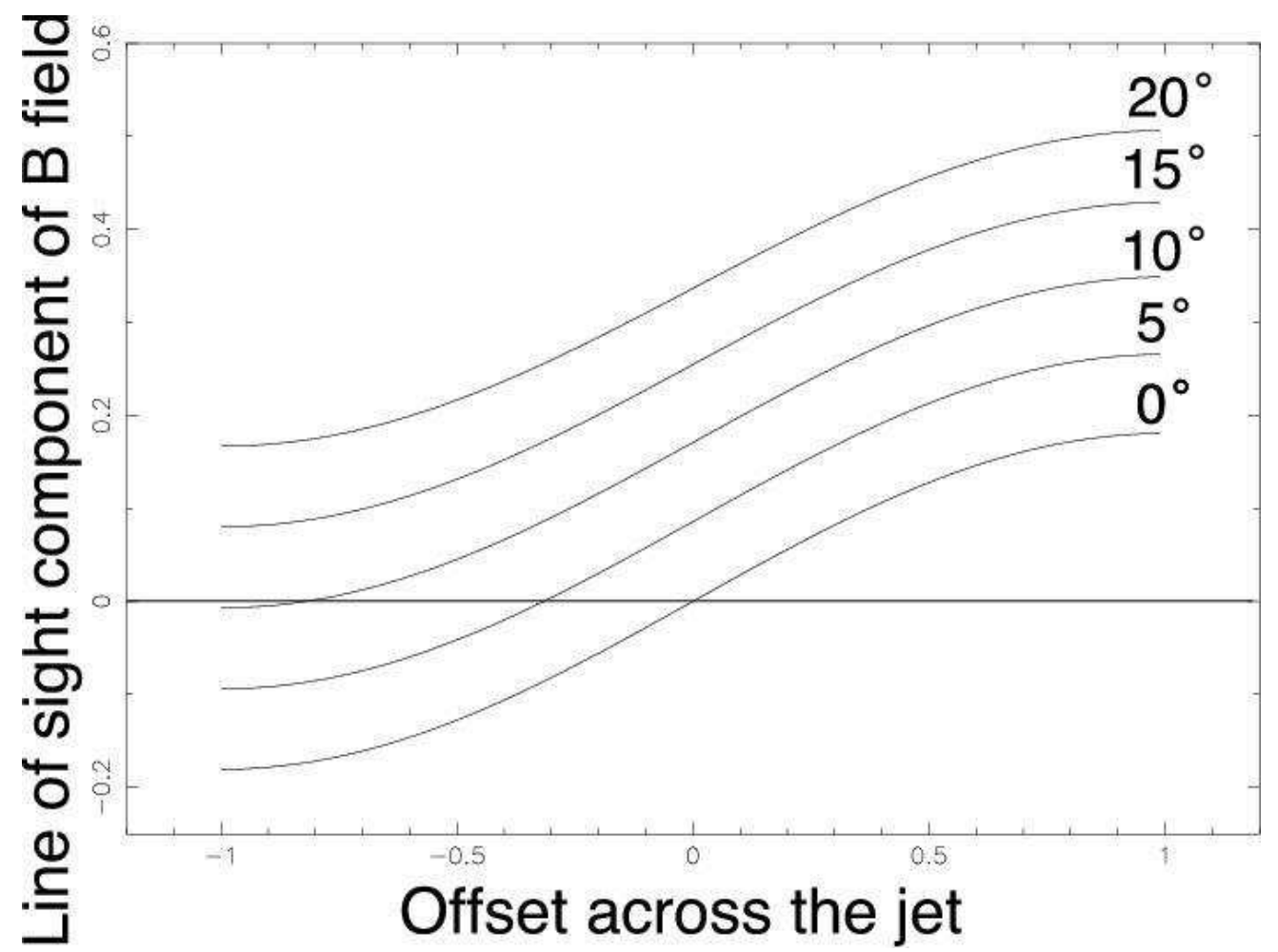

Fig. 4. - Line-of-sight component of the helical magnetic field with pitch angle at $0^{\circ}, 5^{\circ}$, $10^{\circ}, 15^{\circ}$ and 20 . ${ }^{\circ}$ The viewing angle of the helical magnetic field is $10.4^{\circ}$, which is same as the upper limit of the viewing angle derived from the Doppler analysis. Vertical and horizontal scales are normalized by the strength of the magnetic field and the width of the jet, respectively. 